\title{
University of Tokyo Nano Satellite Project "PRISM"
}

\author{
By Mitsuhito KOMATSU and Shinichi NAKASUKA
}

Department of Aeronautics and Astronautics, the University of Tokyo, Tokyo, Japan

\author{
(Received May 2nd, 2008)
}

\begin{abstract}
ISSL is now developing a new super small satellites for Earth remote sensing, "PRISM". This satellite has a unique optics system with flexible extensible boom, aiming at 30m ground resolution. Super small remote sensing satellite can be used for the low-cost global Earth observation missions. To achieve such high ground resolution with flexible telescope, it has some compensation strategies against structural errors or dynamic movement. This satellite will be launched with GOSAT by H-IIA rocket in 2008, and we have finished FM development phase. This paper describes its key concepts and features of PRISM, as well as the problems we encountered and the methodologies for project management by university students.
\end{abstract}

Key Words: Remote sensing, Extensible boom, Management for students' space project

\section{Introduction}

Since 1999, Intelligent Space Systems Laboratory (ISSL) of University of Tokyo has been engaged in super small satellite projects led by university students. In June 2003, our first pico-satellite CubeSat XI-IV [sai-four] (Fig.1) was launched from Plesetsk Cosmodrome, Russia. $\mathrm{XI}-\mathrm{IV}$ is still alive and working more than four years as of April 2008. We finished all planned operations \& experiments with this satellite, and today we permit some amateur radio collaborators to operate the satellite. This "HAM service" gained the support of a lot of people who have interests in amateur radio and satellite communication.

Our second satellite, CubeSat XI-V (Fig.2), was launched from Plesetsk by Cosmos-3M Launcher in October 2005. XI-V is also still working well and we keep up carrying out its operation.

Main Mission of these CubeSats is technology demonstration of basic bus systems for super small satellite with Commercial-Off-The-Shelf products. This enables us to reduce the total cost of development drastically. XI-IV and $\mathrm{XI}-\mathrm{V}$ also have a CMOS image sensor, and they have been sent lots of Earth images (Fig.3). Although this function was additional, advanced mission, XI showed possibilities of the super small satellites being used as an application satellite in the near future.

After the completion of CubeSat projects, we started the design of our new small satellite with a practical mission. The code name of the satellite is "PRISM", which stands for "Pico-satellite for Remote-sensing and Innovative Space Missions" (Fig.4).

In PRISM project, we have selected remote sensing as the main mission. $1^{\text {st }}$ reason of the selection is a motivation to propose an innovative Earth observation system with constellation of such super small satellites. Surrey Satellite Technology Ltd (SSTL) has already built small remote sensing satellites, but they are still large compared to our design. On Table 1 these satellites are listed up with PRISM.
This table directly shows that we aim at different market from SSTL satellites.

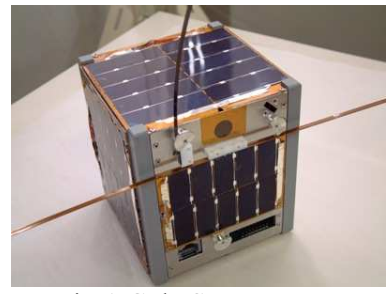

Fig. 1 CubeSat XI-IV

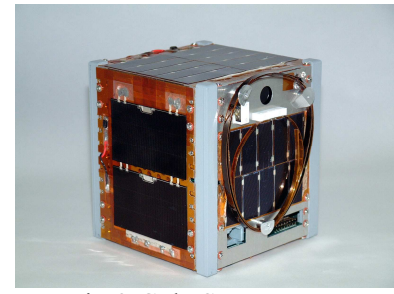

Fig.2 CubeSat XI-V
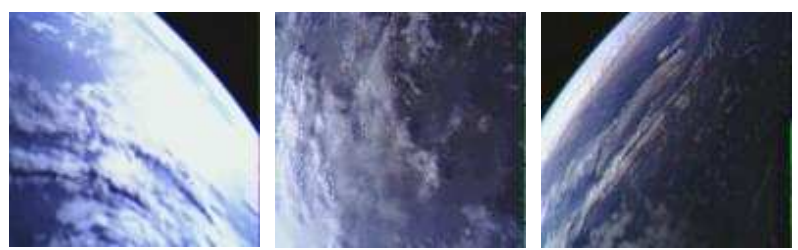

Fig.3 Earth images taken by XI-IV

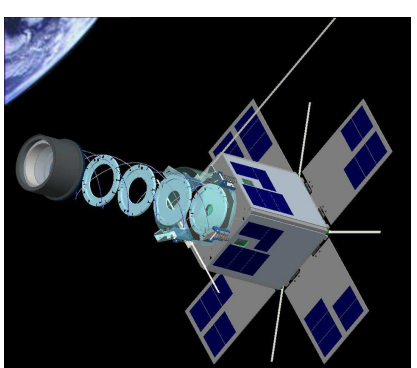

Fig.4 Outlook of PRISM

Table 1. Small Satellites for remote sensing

\begin{tabular}{|c|c|c|c|}
\hline Year & Satellite & Resolution[m] & Mass[kg] \\
\hline 1991 & UoSat-5 & 2000 & 48 \\
\hline 1999 & UoSat-12 & 10 & 312 \\
\hline 2002 & AISat & 32 & 90 \\
\hline 2004 & TopSat & 2.5 & 110 \\
\hline $\mathbf{2 0 0 8}$ & PRISM & $\mathbf{3 0}$ & $\mathbf{8}$ \\
\hline
\end{tabular}


In this paper, we introduce some key concepts and technologies in the design of PRISM, technical issues as well as some management topics. Section 2 describes mission statement with main concepts, followed by specification information of the satellite. In section 3 , we concretely argue about its most distinctive property $(=$ flexible extensible boom) and its property, and feasibility of our mission. In section 4 we introduce bus system of PRISM. The latter half of this paper, started with Section 5, is a little different from former because it argues about the methodologies of project management by university students. We regard this as one of main "component" of university students' satellite.

\section{Overview}

\subsection{Mission}

In order to acquire high resolution Earth images, conventional remote sensing satellites have to contain optical system with multiple lenses or reflection mirrors to fold the long focal length within their structure. Such system requires stiffness of the structure, which induces the increase of mass.

We offer a totally different methodology in PRISM optics system to solve such technological problem.

The most distinctive property of this satellite is, as shown in Fig.4, the mechanical structure of optics system. Fig. 5 is an enlargement of this optical boom.

In order to reduce the total size of telescope, boom of PRISM has an extensible structure (Fig.5). The structure consists of flexible materials, so that they can extend by only the internal elastic force. It doesn't need any mechanical actuators. These features of flexible extensible boom enable us to design a very compact, light-weight optics system (Fig.6). This aspect fits well to the concept of super small satellites. Therefore the main mission of PRISM is as following:

"Technology demonstration of compact optics system with flexible extensible boom by achieving high-resolution remote sensing with a super small satellite"

It is the most important for us to reduce and control the structural errors or errors due to dynamic movement of the boom. To solve these technological problems, PRISM has some error-compensation mechanism described in section 3 .

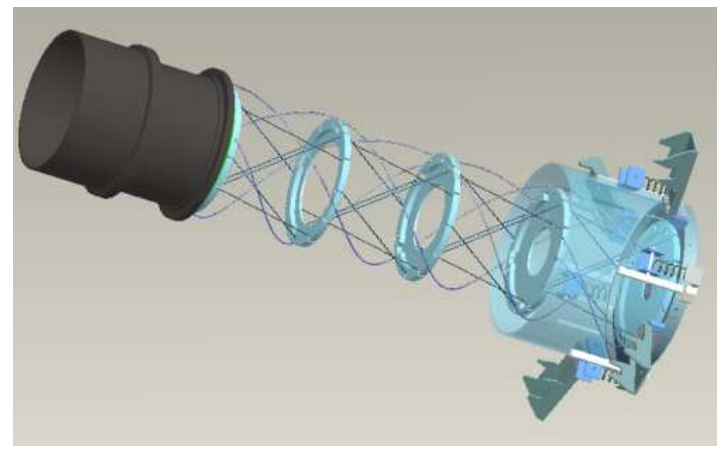

Fig.5 Flexible and extensible boom

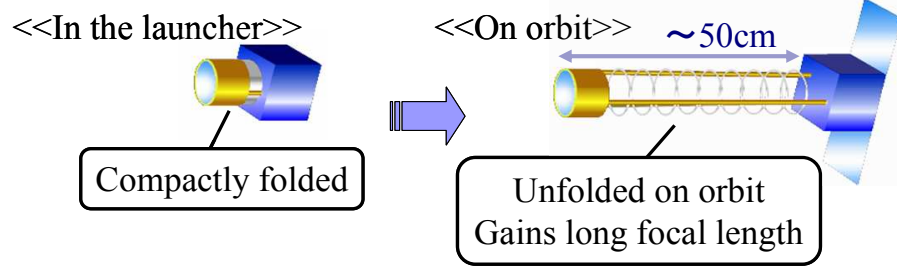

Fig.6 Basic concept of extensible boom

\subsection{Specification}

PRISM is a nano-scale remote sensing satellite. Its design architecture $\&$ methodologies are mainly based on CubeSat XI series; cubic main structure, monopole \& dipole antennae, unfolding mechanism, or rocket interface. By reusing these bus technologies we can gain both of efficiency of development and reliability of these systems.

Table. 2 shows the primal specification of PRISM.

Table 2. Specification of PRISM

\begin{tabular}{|c|c|c|}
\hline \multicolumn{1}{|c}{ Table 2. Specification of PRISM } & Notes \\
\hline Item & Value & $\begin{array}{c}\text { Boom } \\
\text { folded }\end{array}$ \\
\hline Total mass & $192 * 192 * 400\left[\mathrm{~mm}^{3}\right]$ & \\
\hline Orbit & $\begin{array}{c}\text { Sun-Synchronous LEO } \\
\text { Altitude } 660 \mathrm{~km}\end{array}$ & \\
& Local time 13:00 & \\
\hline Attitude & 3-axis magnetic sensor, & \\
Sensors, & Sun sensor $\times 5$, & \\
Controller & 3-axis magnetic torquer & \\
\hline CPU & SH7145F (Renesas & \\
& Technology) & \\
& H8-3048F(Renesas & \\
& Technology) & \\
& PIC-16F877(Microchip) & \\
\hline
\end{tabular}

\section{Optics System Design}

Table 3 summarizes the design property of our optics system.

Table 3. PRISM Optics System

\begin{tabular}{|c|c|c|}
\hline Item & Value & Notes \\
\hline Architecture & Refractive & \\
\hline Lens & $\begin{array}{c}\text { Fluorite apochromat } \\
\text { lenses }\end{array}$ & \\
\hline Aperture & $90[\mathrm{~mm}]$ & \\
\hline Focal length & $500[\mathrm{~mm}]$ & \\
\hline F & 5.6 & $\begin{array}{c}\text { Lens }+ \\
\text { boom }\end{array}$ \\
\hline Total mass & $2[\mathrm{~kg}]$ & \\
\hline Camera & $\begin{array}{c}\text { IBIS-5A (Fill Factory) } \\
\text { CMOS } 1.3 \mathrm{M} \text { Pixel }\end{array}$ & \\
\hline $\begin{array}{c}\text { Ground } \\
\text { Resolution }\end{array}$ & $10[\mathrm{~m}]$ per pixel & \\
\hline FOV & $6.51[\mathrm{~km}] * 6.51[\mathrm{~km}]$ & \\
\hline
\end{tabular}

We chose a refractive system design, not a reflective one. Reflective system requires severe alignment accuracy of mirrors or lenses. On the other hand, in refractive system chromatic aberration may occur. We compensate 
this by using fluorite apochromat lenses. These lenses are coated with UV shield because they will be exposed to $\mathrm{UV}$ and radiation ray in space. We also carried out UV and radiation test to these devices.

Flexible extensible boom is a key component for our mission (Fig.7). GFRP frame plays a role of coiled spring, which pushes Lens or buffle plates out when the structure is unfolded and extended. Some threads connect buffle plates each other constraining each distance between them, and they determine the total length of the boom. They also determine relative position, tilt angle of the lens toward the image sensor.

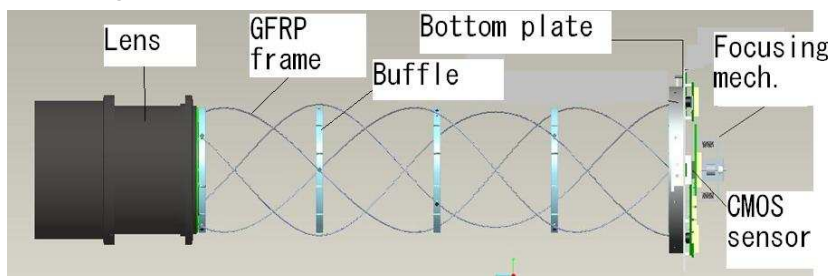

Fig.7 Components of Boom

Compensation of structural error of the boom is the most distinctive technology for our project. We made 4 solutions about this as following:

1. dynamically stable structure (analytic model)

We made linear analytic model of the structure, calculating stability against tiny error (i.e. inequality of thread lengths or that of elastic coefficients of GFRP) and found a dynamically static point of design.

2. static error compensation

This is a way to compensate inequality of thread lengths. A few of thin spacers are inserted between buffle plate and stopper of the thread. We can also insert such spacers between the root of boom and bottom plate, so that we can gain offset of total length of boom. The accurate length of the boom or each thread is measured by laser or images obtained by the experiment of whole system.

3 . extension shock absorber

Boom structure and main cubic body of PRISM is jointed by only 4 springs. These springs absorb the shock of extension due to inertia of lens. This shock may cause damage or errors of the boom structure without such a shock absorber.

4. focusing mechanism

Fig. 8 shows design of our focusing mechanism. Image sensor board is pushed by stepping motor while it's pulled by springs (Fig.8). The board is guided by linear rail and can move straightforwardly. Thus the relative position of the image sensor toward the lens can be dynamically controlled, and then we can compensate remained structural error of the boom.

In order to guarantee the feasibility of compensation of structural errors by these strategies, we carried out micro-gravity experiment. With this experiment we obtained estimation value of static offset of boom length under micro-gravity environment from $1 \mathrm{G}$ environment. We also acquired the STD error of boom total length by the same experiment, which turned out to be much smaller than the range of compensation of focusing mechanism.

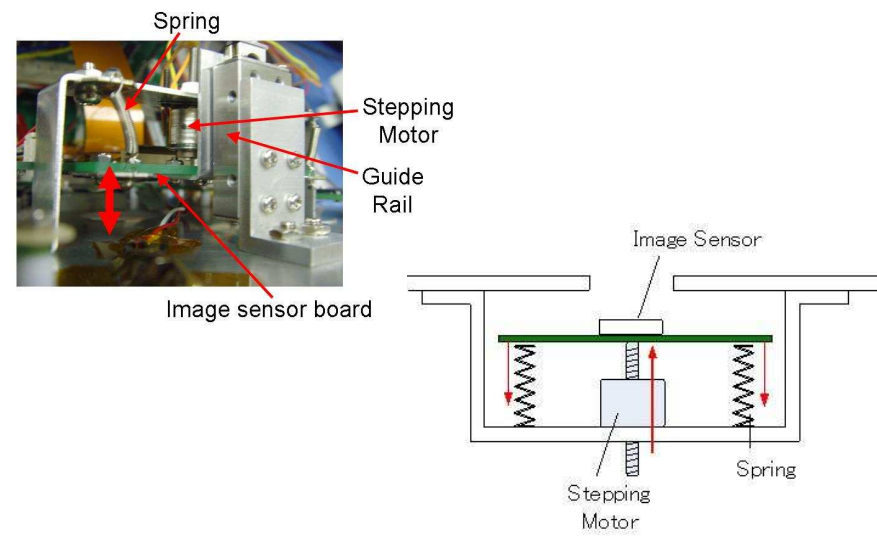

Fig. 8 Focusing mechanism

\section{Bus System of PRISM}

Bus system of PRISM consists of following subsystems:

- Power

- Communication

- Command and Data Handling (C\&DH)

- Attitude Determination and Control System (ADCS)

- Thermal \& Structure Subsystem

\subsection{Power}

Power subsystem takes charge of electric power management and health monitoring of all subsystem. Processors in PRISM generate $50 \mathrm{~Hz}$ pulses to show their aliveness and send them to each other. When Power control processor detects abnormal behavior of others, it shut down \& reset the power line of them.

Power subsystem also has current limiter circuit for each subsystem. They prevent devices of other subsystems from burning out by SEE.

Furthermore, power subsystem includes $\mathrm{CW}$ beacon transmitter. The reason why this module is included in power subsystem is described in section 5 .

\subsection{Communication}

PRISM communication subsystem consists of a Tx module and 2 Rx modules.

Tx module has 2 radio modules, AFSK1200bps / GMSK9600bps. Specification of these radio modules described in Table 4 . These radio was developed by a Japanese radio manufacturer. AFSK module is mainly used for downlink of house-keeping data, such as temperature sensor or current sensors. On the other hand, with GMSK module we mainly send image data. GMSK module has 8 times better communication rate than AFSK module, and the image data is the largest data in the telemetry data of PRISM.

PRISM is equipped with 2 receivers. One of them is used for mission control by administrators of the satellite (Rx-Main), while the other is a backup and will be also used for additional missions of amateur radio service 
(Rx-Sub). The uplink frequency and operation command of Rx-Sub is published after we have finished the basic function of all system. Rx-Sub also has EEPROM and it can store the message from amateur radio operators.

Table 4 Specification of Radio Module

\begin{tabular}{|l|l|l|l|l|}
\hline Modulation & AFSK & GMSK & CW & Rx \\
\hline Freq. band & $460 \mathrm{Mhz}$ & $430 \mathrm{MHz}$ & $43 \mathrm{MHz}$ & $144 \mathrm{MHz}$ \\
\hline $\begin{array}{l}\text { Output } \\
\text { Power }\end{array}$ & $800 \mathrm{~mW}$ & $2-3 \mathrm{~W}$ & $100 \mathrm{~mW}$ & --- \\
\hline Data rate & $1200 \mathrm{bps}$ & $9600 \mathrm{bps}$ & $50 \mathrm{wpm}$ & $1200 \mathrm{bps}$ \\
\hline Antenna & Dipole & Turnstile & Dipole & Monopole \\
\hline
\end{tabular}

\subsection{Command and Data Handling (C\&DH)}

Command and Data Handling subsystem consists of main processor "Renesas-SH7145F", some RAMs, ROMs, an $\mathrm{A} / \mathrm{D}$ converter and Real time clock (RTC). This subsystem has following functions:

1. Management of inter-subsystem issues among C\&DH, ADCS and Optics

C\&DH, ADCS and Optics subsystem coexist within one processor, $\mathrm{SH} 7145 \mathrm{~F}$, so that we can reduce total system size. Then C\&DH subsystem controls processing thread and manages processing resources among these subsystems. For example, when uplink commands to shoot an image from GS come, C\&DH enables optics to do their work, and disables again after finishing that work.

2. Interpreting and processing uplink commands

Uplink commands from the ground station are decoded by RxTNC and are sent to each processor via Controller Area Network.

3. Storing sensor telemetry data for downlink

Acquiring A/D conversion data by using A/D converter, C\&DH stores every sensor data in PRISM temperature, output current or voltage of solar cells, battery voltage, gyroscopes and magnetometers, and so on. C\&DH also add error detection \& correction code with these telemetry data, which enables us to correct SEU errors occurred in those data.

4. Management of processing ADCS \& Optics functions

As described above, functions of C\&DH, ADCS and Optics subsystems are implemented in one chip. To realize real-time task management of these functions, once we examined to utilize Real-time OS (micro-ITRON). But finally we chose our original task management software because RTOS conceal internal software flow \& task management layer and user can't predict what happens when SEU occurs.

In the future, the structures of software in the spacecraft will be more complicated and some kind of operating system will be essential. And the OS for such application field will have to have a sort of "process traceability", which can simulate the sequence of process affected by the single event failure and show the results of them.

5. Real time clock management

C\&DH has Real time clock (RTC), and is used for 3-dimensional position with Earth Centered Inertial Coordinate. Orbit information will be sent from GS as 2 line elements format. ADCS can calculate its position from RTC output and orbit information.

6. On-orbit reconfiguration of the program with uplink command

We implemented reprogramming function on orbit to $\mathrm{C} \& \mathrm{DH}$ as an advanced mission. This function enables us to correct errors in code of $\mathrm{SH}$, as well as to improve control algorithms of ADCS.

\subsection{Attitude Determination and Control System (ADCS)}

ADCS controls the attitude of PRISM to satisfy mission requirements from optical system. This subsystem has following functions:

(1) Steering the lens at Earth

In space, the gravity gradient torque affects spacecrafts. For this reason, whether the lens or the satellite body steers at Earth and nominally stabilizes. The lens may steer at Earth first, and vice versa. If PRISM has stabilized upside down, ADCS takes charge of its remediation.

(2) Stabilization of attitude

The extensible boom works as a gravity gradient boom, which lessen the burden of attitude control. However, attenuating the vibration will be still required. Remaining magnetic moment may also cause the perturbation of its attitude, because the mass or values of inertia matrix of PRISM are relatively small in comparison with the figure of its magnetic sensitivity. With ADCS, PRISM can stabilize the oscillation of flexible boom.

ADCS has magnetometers, gyros, sun sensors as attitude determination sensors, and magnetorquers as actuators.

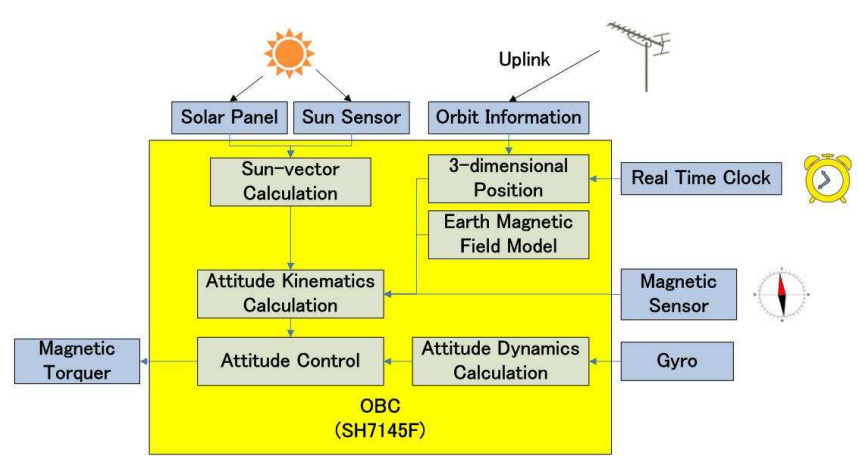

Fig. 9 ADCS system block diagram

\subsection{Thermal \& Structure subsystem}

Thermal \& Structure subsystem integrates all hardware into satellite body, designing appropriate thermal properties against the space environment. They also take charge of development of the rocket interface of shown in Fig. 10. 


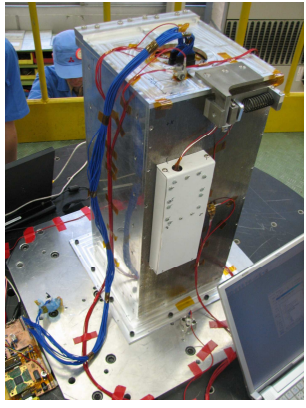

Fig. 10 Rocket Interface box of PRISM

\section{Project Management}

\subsection{Circumstances}

PRISM project started in 2002. We first made conceptual design, and completed the first BBM (Bread Board Model) in February 2003. Then the project had suspended because of the launch \& operation of XI-IV and XI-V or other projects. We restored our project in 2006 spring, but almost all of members changed places with new students. Succession of the concept of design between them is not sufficient, so we had to restart from the conceptual design \& BBM. We finished this and developed Engineering Model in 2007, and passed the judging committee of safety by JAXA. The Flight model had been developed from January to May this year, and is now under the environmental test. The date of launch campaign has not been decided, but we will be ready for launch by July.

\subsection{Organization of project team}

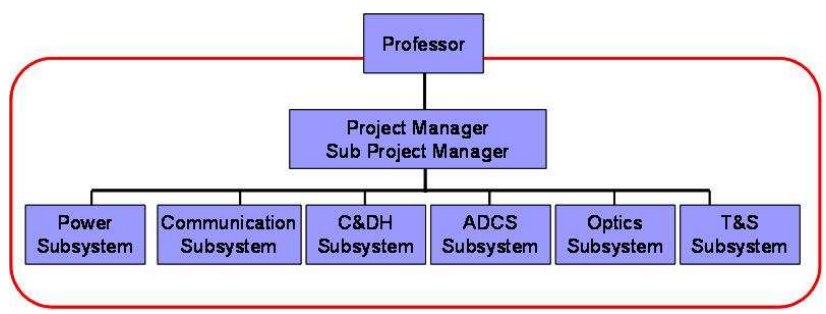

Fig.11 Project organization

Fig.11 shows the organization of our project. All of members except professor are graduate/ under-graduate students. From CubeSat projects, these super small satellite projects have been led by students in ISSL.

PRISM project consists of following six subsystems:

- Optics

- Attitude Determination and Control System (ADCS)

- Command and Data Handling (C\&DH)

- Communication

- Power

- Thermal \& Structure design

Currently 18 students (graduate or under-graduate) are engaged in the project.

System for information exchange among members is so significant for project management. Now we have short meeting everyday, for real-time information exchange among members or subsystems. We also have subsystem meetings \& leader meeting, and there we arrange each task of members and build schedule \& plan. With these meetings we can also share problems of each member and watch on degree of progress of their activities.

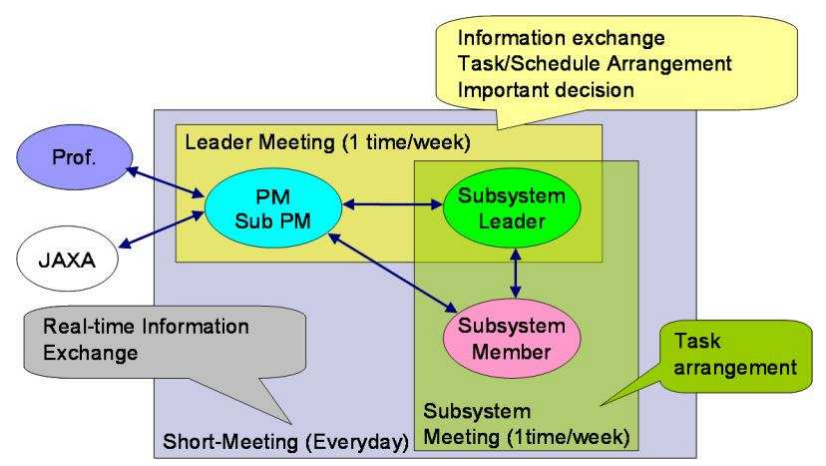

Fig.12 Information Exchange among members

\subsection{Document management system}

Documents describing project information are so significant, because student members change places with new students every 2 or 3 years. We have to store the engineering knowledge, design concepts of satellites, reasons of choice of components, reflection on some failures, notes or instructions of handling machines \& tools, and so on.

We have developed original document management system with database. User can also search documents by free keywords, authors or other options.

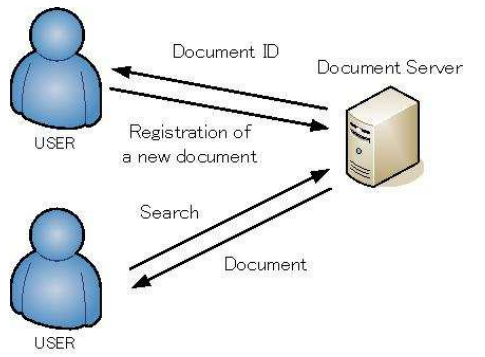

Fig.13 Document Management System

\subsection{Component Dependency Diagram \\ 5.3.1. Basic idea of CDD}

Especially on the design phase of satellite, a lot of conflicts occur on resources such as volume, mass, electric power, costs and so on. With such cases, we have to understand priorities of each component properly, and judge which requirement we should take precedence.

To visualize the priorities of each component in the satellite, we developed "Component Dependency Diagram" (CDD). Fig.14 is an example of CDD. This diagram shows that component $\mathrm{C}$ doesn't work unless both of components A and B work perfectly. Generally speaking, components $\mathrm{A}$ and $\mathrm{B}$ are more important and have more priorities than $\mathrm{C}$ on this point of view.

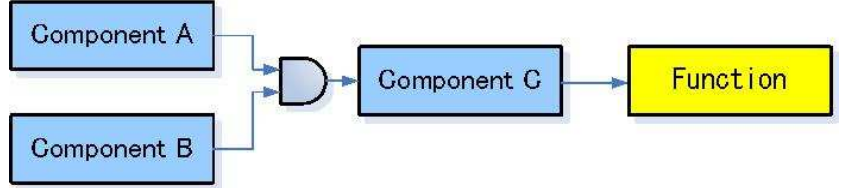

Fig.14 Component Dependency Diagram 


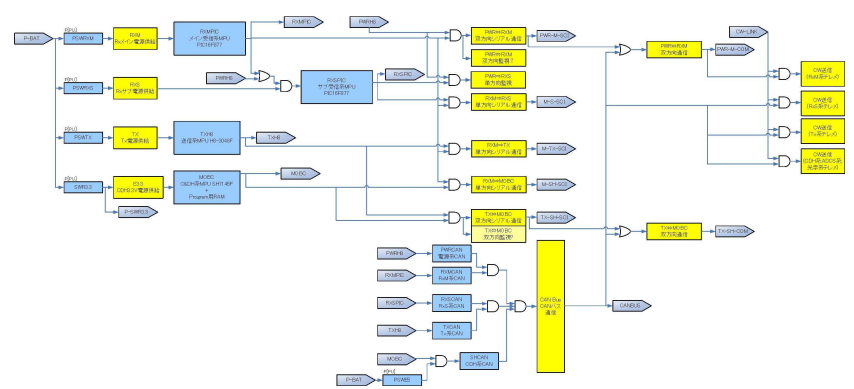

Fig.15 A part of CDD of PRISM

CDD also tells us a proper procedure of development. In general, we should develop or confirm the property of components A,B before component $\mathrm{C}$. This guideline can be applied to the test phase of integrated systems. Following is one of the successful cases of application of CDD.

\subsubsection{An example of CDD application to system design of PRISM}

$\mathrm{CW}$ transmission is one of the most basic functions in our satellite, and all of the components required for the function have the highest priorities. They have to be awake (i.e. eclectically power-on) during almost all of its mission life. Making this architecture as simple as possible is so important because it can reduce their power consumption, and it can also make the system more reliable.

In June 2007, we analyzed the system architecture and described the mutual dependencies among components in the system. Fig.16 is a part of the results (Some components are not described for not confusing). Red lines show power supply lines, and blue ones are for control.

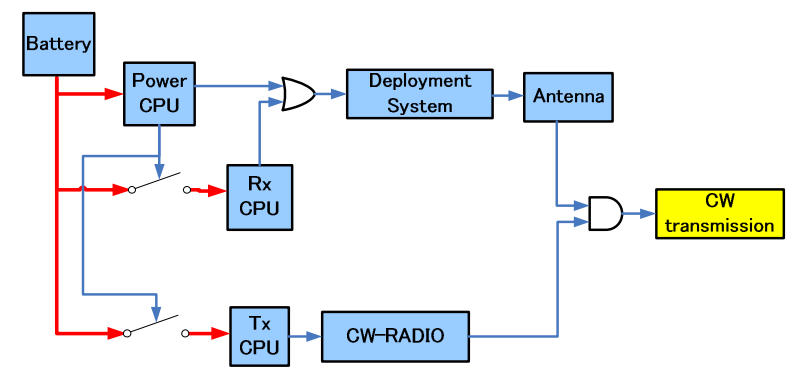

Fig.16 CW system architecture (ver. Jun. 2007)

After we finished analyzing the system in July 2007, a member of our project team saw fig. 16 and found that we could make it simpler by controlling $\mathrm{CW}$ radio module directly from power CPU. The newly designed architecture is described in Fig.17. With this design change, the reliability of CW transmission system of PRISM became independent from that of Tx CPU. Total electric power consumption is also minimized.

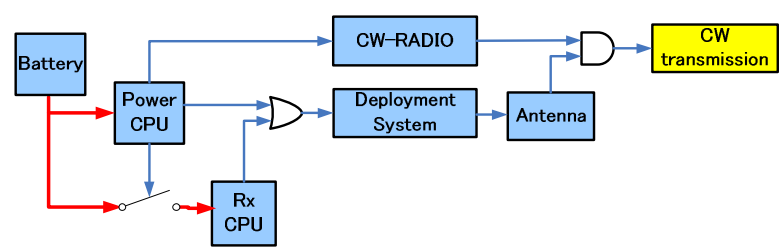

Fig. 17 CW system architecture (ver. Jul. 2007)

\section{Conclusions}

In this paper, the outline of PRISM project is presented. PRISM is a next generation of Japanese super small satellites with advanced missions and attractive features, deployment system of boom, antennae and solar array panels, active attitude control system, high data communication as well as the reinforced bus technology verification. We made a lot of devices in the project management, and they can be reused by following projects.

\section{References}

\section{References}

1 Y. Nakamura, T. Eishima, M. Nagai, R. Funase, A. Enokuchi, K. Nakada, Y. Cheng, E. T. Takei, T. Funane, F. Sasaki, Y. Nojiri, T. Yamamoto, E. Nagayama and $S$. Nakasuka,"Demonstration of High-Resolution Remote Sensing by University of Tokyo's Next Generation Pico-Satellite "PRISM", $24^{\text {th }}$ International Symposium on Space Techonology and Science

2 N. Miyamura, T. Eishima, Y. Nakamura, R. Funase, A. Enokuchi, Y. Hatsutori, T. Funane, Y. Nojiri, F. Sasaki and S. Nakasuka, "HIGH RESOLUTION EXTENSIBLE TELESCOPE FOR UNIVERSITY OF TOKYO'S REMOTE SENSING Nano-SATELLITE "PRISM"" $25^{\text {th }}$ International Symposium on Space Technology and Science.

3 Y.Sato, S.K.Kim, Y.Kusakawa, K.Shimizu, T.Tanaka, M.Komatsu, Y.I.Yun, C.Lambert and S.Nakasuka, "Extensible Flexible Optical System for Nano-Scale Remote Sensing Satellite "PRISM" ", The 26th International Symposium on space Technology and Science

4 PRISM web site: http://www.space.t.u-tokyo.ac.jp/prism/

5 CubeSat web site: $\underline{\text { http://www.space.t.u-tokyo.ac.jp/cubesat/index-e.html }}$ 